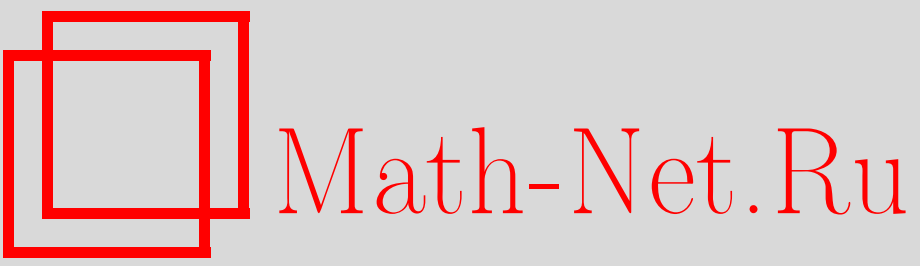

М. М. Глухов, Об использовании групп классов идеалов квадратичных полей для построения криптографических систем с открытым ключом, Maтем. вопр. криптогр., 2010, том 1, выпуск 1, 23-54

DOI: https://doi.org/10.4213/mvk3

Использование Общероссийского математического портала Math-Net.Ru подразумевает, что вы прочитали и согласны с пользовательским соглашением

http://www. mathnet.ru/rus/agreement

Параметры загрузки:

IP : 54.81 .137 .203

26 апреля 2023 г., 04:10:09 


\title{
Об использовании групп классов идеалов квадратичных полей для построения криптографических систем с открытым КЛючом
}

\author{
М. М. Глухов \\ Академия криптографии Российской Федерации, Москва
}

Получено 22.IV.2010

В статье приводится краткий обзор результатов по криптосистемам, основанным на группах классов идеалов мнимых и вещественных квадратичных полей.

Ключевые слова: квадратичное поле, кольцо целых алгебраических чисел, группа классов идеалов, редуцирование идеалов, система распределения ключей, проблема дискретного логарифмирования.

On application of class groups of ideals of quadratic fields to the construction of public key cryptosystems

M. M. Gluhov

Academy of Cryptography of Russian Federation, Moscow

Abstract. The paper contains a brief survey on cryptosystems based on imaginary and real quadratic fields.

Key words: quadratic field, ring of integer numbers of quadratic fields, class group, algorithmic reduction of ideals, key exchange system, discrete logarithm problem.

Citation: Mathematical Aspects of Cryptography, 2010, vol. 1, no. 1, pp. $23-54$ (Russian). 


\section{Введение}

К настоящему времени опубликовано большое число статей, посвященных построению и анализу криптографических систем с открытым ключом, основанных на использовании групп классов идеалов колец целых алгебраических чисел и немаксимальных порядков расширений поля рациональных чисел $\mathbf{Q}$ (см. [12-73]).

В классической теории алгебраических чисел более полно изучены квадратичные расширения поля $\mathbf{Q}$. Поэтому, в основном, только такие расширения и предлагается использовать для построения криптографических систем. Криптографическая стойкость таких систем обеспечивается сложностью таких проблем теории чисел как проблема вычисления порядков групп классов идеалов, проблема дискретного логарифмирования в указанных группах и проблема главных идеалов. В связи с этим появилось много публикаций по построению алгоритмов решения указанных проблем в различных (не обязательно квадратичных) кольцах алгебраических чисел и по оценкам сложности предлагаемых алгоритмов. В итоге сложилось оригинальное направление криптографических исследований, представляющее как теоретический, так и практический интерес.

В данной работе дается краткий обзор основных результатов этого направления. При асимптотических оценках сложности алгоритмов используются общепринятые обозначения:

$$
L_{n}(e, c)=\exp \left(c(\log n)^{e}(\log \log n)^{1-e}\right), n \in N, c \in R, c>0,0 \leq e \leq 1,
$$

и его сокращение $L_{n}(c)$ при $e=1 / 2$.

\section{1. Необходимые сведения из общей теории алгебраических чисел}

Излагаемый ниже материал можно найти в [1-11].

Комплексное (в частности, действительное) число $\theta$ называется алгебраическим над полем $\mathbf{Q}$, или просто алгебраическим, если оно является корнем некоторого многочлена над $\mathbf{Q}$. При этом унитарный многочлен наименьшей степени с корнем $\theta$ называется минимальнымм многочленом числа $\theta$ и обозначается через $m_{\theta}(x)$. Степень этого многочлена называется степенью числа $\theta$, а также степенью расширения $\mathbf{Q}(\theta)$ поля $\mathbf{Q}$ с помощью числа $\theta$, что обозначается через $n=[\mathbf{Q}(\theta): \mathbf{Q}]$. Многочлен $m_{\theta}(x)$ неприводим над $\mathbf{Q}$ и имеет в поле комплексных чисел $\mathbf{C}$ ровно $n$ различных корней $\theta_{1}=\theta, \theta_{2}, \ldots, \theta_{n}$. Все они называются числами, сопряженными с $\theta$. Если среди них $s$ действительных и $t$ пар мнимых сопряженных, то упорядоченная пара чисел $(s, t)$ называется сигнатурой поля $\mathbf{Q}(\theta)$. 
Любой элемент $\alpha$ поля $\mathbf{Q}(\theta)$ однозначно представляется в виде

$$
\alpha=a_{0}+a_{1} \theta+\ldots+a_{n-1} \theta^{n-1}, a_{0}, a_{1}, \ldots, a_{n-1} \in \mathbf{Q} .
$$

Следовательно, набор элементов $\left(1, \theta, \ldots, \theta^{n-1}\right)$ является базисом поля $\mathbf{Q}(\theta)$ как векторного пространства над $\mathbf{Q}$. Любой базис этого пространства называют также базисом поля $\mathbf{Q}(\theta)$.

Число $\alpha \in \mathbf{Q}(\theta)$ называется цельмм алгебраическим числом, или целым числом поля $\mathbf{Q}(\theta)$, если $m_{\alpha}(x) \in \mathbf{Z}[x]$. Множество $\mathbf{Q}_{0}(\theta)$ всех целых чисел поля $\mathbf{Q}(\theta)$ является кольцом. Для любого числа $\alpha$ из $\mathbf{Q}(\theta)$ существует такое число $r \in \mathbf{Z}$, что $r \alpha \in \mathbf{Q}_{0}(\theta)$. В связи с этим при изучении поля $\mathbf{Q}(\theta)$ можно считать, не теряя общности, что $\theta$ является целым алгебраическим числом. Далее $\theta$ будем считать фиксированным и, для краткости, поле $\mathbf{Q}(\theta)$, кольцо $\mathbf{Q}_{0}(\theta)$ и его мультипликативную группу будем обозначать соответственно через $\mathbf{K}, \mathbf{K}_{0}$ и $\mathbf{K}_{0}{ }^{*}$.

При изучении поля К существенно используются его изоморфизмы над $\mathbf{Q}$ в поле комплексных чисел $\mathbf{C}$. Любой такой изоморфизм определяется образом элемента $\theta$. Изоморфизм, отображающий $\theta$ в $\theta_{i}$, обозначим через $\sigma_{i}, i=1, \ldots, n$. Числа

$$
\mathrm{N}(\alpha)=\sigma_{1}(\alpha) \ldots \sigma_{n}(\alpha) \quad \text { и } \operatorname{Tr}(\alpha)=\sigma_{1}(\alpha)+\ldots+\sigma_{n}(\alpha)
$$

называются соответственно нормой и следом числа $\alpha$. Функции $\mathrm{N}(x)$ и $\operatorname{Tr}(x)$ обладают следующими свойствами.

Для любых $\alpha, \beta \in \mathbf{K}$ :

$$
\mathrm{N}(\alpha), \operatorname{Tr}(\alpha) \in \mathrm{Q}, \mathrm{N}(\alpha \beta)=\mathrm{N}(\alpha) \mathrm{N}(\beta), \operatorname{Tr}(\alpha+\beta)=\operatorname{Tr}(\alpha)+\operatorname{Tr}(\beta) .
$$

Для любого $\alpha \in \mathbf{K}_{0}$ справедливы соотношения $\mathrm{N}(\alpha), \operatorname{Tr}(\alpha) \in \mathbf{Z}, \alpha \mid \mathrm{N}(\alpha)$ (число $\alpha$ делит $\mathrm{N}(\alpha)$ в кольце $\left.\mathbf{K}_{0}\right), \alpha \in \mathbf{K}_{0}{ }^{*} \Leftrightarrow|\mathrm{N}(\alpha)|=1$.

Строение группы $\mathbf{K}_{0}{ }^{*}$ описывается классической теоремой Дирихле, согласно которой $\mathbf{K}_{0}^{*}$ представляется в виде прямого произведения некоторой конечной группы $\Gamma_{m}$ всех комплексных корней $m$-й степени из 1 и $s+t-1$ бесконечных циклических групп.

Элементы группы $\mathbf{K}_{0}^{*}$ называют обычно единицами поля $\mathbf{K}$, а образующие бесконечных циклических сомножителей группы $\mathbf{K}_{0}{ }^{*}-$ фундаментальными единицами.

Обозначим через $\mathbf{I}\left(\mathbf{K}_{0}\right)$ множество всех идеалов кольца $\mathbf{K}_{0}$. Известно, что каждый идеал $A \in \mathbf{I}\left(\mathbf{K}_{0}\right)$ порождается некоторой конечной системой элементов $v_{1}, \ldots, v_{k}$, что записывается равенством

$$
A=\left(v_{1}, \ldots, v_{k}\right) .
$$


Более того, для каждого идеала кольца $\mathbf{K}_{0}$ существует двухэлементное задание $A=(\alpha, \beta)$, в котором $\alpha \in \mathbf{Z}$. Аддитивная группа $(A,+)$ является свободной абелевой группой ранга $n$. Следовательно, существует такая система элементов $w_{1}, \ldots, w_{n} \in A$, при которой любой элемент $\alpha \in A$ однозначно представляется в виде

$$
\alpha=z_{1} w_{1}+\ldots+z_{n} w_{n},
$$

где $z_{1}, \ldots, z_{n} \in \mathbf{Z}$. Любая такая система называется $\mathbf{Z}$-базисом идеала А, что обозначается записью $A=\left[w_{1}, \ldots, w_{n}\right]$. Группа $(A,+)$ является подгруппой конечного индекса в группе $\left(\boldsymbol{K}_{0},+\right)$, ее индекс называется нормой идеала $A$ и обозначается через $\mathrm{N}(A)$. Таким образом,

$$
\mathrm{N}(\mathrm{A})=\left[\mathbf{K}_{0}: \mathrm{A}\right] .
$$

Идеал $A$ называется главным, если он порождается одним элементом. Если $A=(\alpha)$, то $\mathrm{N}(A)=|\mathrm{N}(\alpha)|$. В общем же случае норма идеала $A$ выражается через дискриминанты идеала $A$ и поля К.

Дискриминантом любой системы из $n$ элементов $w_{1}, \ldots, w_{n} \in \mathbf{K}$ называется число

$$
d\left(w_{1}, \ldots, w_{n}\right)=\Delta\left(w_{1}, \ldots, w_{n}\right)^{2},
$$

где

$$
\Delta\left(w_{1}, \ldots, w_{n}\right)=\operatorname{det}\left(\sigma_{i}\left(w_{j}\right)\right) .
$$

Если $w_{1}, \ldots, w_{n}$ есть $\mathbf{Z}$-базис идеала $A$, то $d\left(w_{1}, \ldots, w_{n}\right)$ называется дискриминантом идеала $A$, и называется дискриминантом поля $\mathbf{K}$, если $A=\mathbf{K}_{0}$. В последнем случае он обозначается через $d(\mathbf{K})$, или, просто, $d$, если поле $\mathbf{K}$ фиксировано. Заметим, что величина дискриминанта идеала не зависит от выбора его $\mathbf{Z}$-базиса. Для любого ненулевого идеала $A$ имеет место равенство

$$
\mathrm{N}(A)=|\sqrt{d(A) / d}| .
$$

Для идеалов, как и для чисел,

$$
\mathrm{N}(A B)=\mathrm{N}(A) \mathrm{N}(B) .
$$

На множестве всех идеалов любого коммутативного кольца естественным образом определяются операции сложения и умножения идеалов. При этом в мультипликативной полугруппе идеалов строится хорошая теория делимости. А именно, выделяются неразложимые идеалы, которые совпадают с максимальными идеалами и с простыми идеалами в общепринятом смысле, и доказывается основная теорема теории идеалов о том, что в коль- 
це $\mathbf{K}_{0}$ каждый идеал, отличный от нулевого и от $\mathbf{K}_{0}$, представляется в виде произведения простых идеалов и что такое представление единственно с точностью до перестановки сомножителей. Заметим, что множество простых идеалов бесконечно. Точнее, для любого простого числа $p \in \mathbf{Z}$ существуют простые идеалы, содержащие $p$, причем идеалы, содержащие различные простые числа, различны.

Из основной теоремы теории идеалов следует, в частности, что полугруппа идеалов $\left(\mathbf{I}\left(\mathbf{K}_{0}\right)\right.$ не является группой, поскольку в ней обратимым является лишь идеал $\mathbf{K}_{0}$, играющий роль единицы. Однако полугруппу $\left(\mathbf{I}\left(\mathbf{K}_{0}\right)\right.$ очень простым способом можно дополнить до группы, включив в нее так называемые дробные идеалы поля К.

Дробным идеалом поля К называется множество всех чисел вида (1), где $w_{1}, \ldots, w_{k}$ - любые фиксированные числа поля $\mathbf{K}$, а $z_{1}, \ldots, z_{k}$ принимают всевозможные значения из кольца $\mathbf{K}_{0}$. Если при этом имеет место однозначность представления чисел в виде (1), то система $w_{1}, \ldots, w_{k}$ называется базисом дробного идеала. Дробный идеал с одноэлементным базисом называется главнылм. Множество всех дробных идеалов поля К обозначим через $\mathbf{I}(\mathbf{K})$. Заметим, что $\mathbf{I}\left(\mathbf{K}_{0}\right) \subset \mathbf{I}(\mathbf{K})$ и что дробные идеалы из $\mathbf{I}(\mathbf{K}) \backslash \mathbf{I}\left(\mathbf{K}_{0}\right)$ не являются идеалами кольца $\mathbf{K}_{0}$ или поля К. Поэтому без особой договоренности слово «дробный» в названии дробного идеала опускать нельзя. Существует простая связь между идеалами кольца $\mathbf{K}_{0}$ и дробными идеалами поля $\mathbf{K}$. А именно, для каждого дробного идеала $B$ существуют идеал $A$ и целое рациональное число $c \neq 0$ такие, что $A=c B$, т. е. $B=(1 / c) A$. Для дробных идеалов сохраняется основная теорема об идеалах. Каждый дробный идеал $A$, отличный от (0) и $\mathbf{K}_{0}$, однозначно с точностью до перестановки сомножителей представляется в виде

$$
A=P_{1}^{k_{1}} \ldots P_{m}^{k_{m}},
$$

где $P_{1}, \ldots, P_{m}$ - различные простые идеалы, а $k_{1}, \ldots, k_{m}$ - отличные от нуля целые числа (среди которых могут быть и отрицательные). Отсюда следует, что множество $\mathbf{I}(\mathbf{K})$ является группой, разлагающейся в прямое произведение бесконечного числа бесконечных циклических групп, порождаемых простыми идеалами.

Главные дробные идеалы поля $\mathbf{K}$ образуют подгруппу, которую мы обозначим через $\mathbf{I}_{1}(\mathbf{K})$. Факторгруппа $\mathbf{I}(\mathbf{K}) / \mathbf{I}_{1}(\mathbf{K})$ называется группой классов идеалов (или просто группой классов) поля $\mathbf{K}$ и обозначается через $\mathrm{Cl}(\mathbf{K})$. Эта группа конечна, ее порядок называется числом классов идеалов (или просто числом классов) поля $\mathbf{K}$ и обозначается через $h(\mathbf{K})$. В общем случае о величине $h(\mathbf{K})$ и о строении группы $\mathrm{Cl}(\mathbf{K})$ известно очень мало. Точные формулы для $h(\mathbf{K})$ известны лишь в отдельных частных случаях, например, 
для расширений поля $\mathbf{Q}$ степени 2, которые называют квадратичными полями. Так как в работах по использованию групп классов идеалов в криптографии фигурируют главным образом лишь квадратичные поля, то остановимся на этом частном случае подробнее.

\section{2. Квадратичные поля и их группы классов}

Любое квадратичное расширение поля $\mathbf{Q}$ можно представить в виде $\mathbf{Q}(\sqrt{D})$, где $D-$ свободное от квадратов и отличное от 0 и 1 целое число. Это расширение поля $\mathbf{Q}$ называется мнимым, если $D<0$, и реальным, или вещественным, если $D>0$. Любое число $\alpha \in \mathbf{Q}(\sqrt{D})$ однозначно представляется в виде

$$
\alpha=a+b \sqrt{D}, \quad a, b \in \mathbf{Q}
$$

При этом сопряженным с ним числом будет $\alpha^{\prime}=a-b \sqrt{D}$. Если $b \neq 0$, то число $\alpha$ имеет минимальный многочлен $x^{2}-2 a x+a^{2}-b^{2} D$, и потому

$$
\mathrm{N}(\alpha)=a^{2}-b^{2} D \quad \text { и } \operatorname{Tr}(\alpha)=2 a .
$$

Следовательно,

$$
\alpha \in \mathbf{Q}_{0}(\sqrt{D}) \Leftrightarrow a^{2}-b^{2} D, 2 a \in \mathbf{Z}
$$

Отсюда легко получается описание всех целых чисел поля $\mathbf{Q}(\sqrt{D})$ :

1) $\mathbf{Q}_{0}(\sqrt{D})=\{a+b \sqrt{D} ; a, b \in \mathbf{Z}\}$, если $D \neq 1(\bmod 4)$,

2) $\mathbf{Q}_{0}(\sqrt{D})=\left\{\frac{a+b \sqrt{D}}{2} ; a, b \in \mathbf{Z}, a \equiv b(\bmod 2)\right\}$, если $D \equiv 1(\bmod 4)$.

Дискриминант $d$ поля $\mathbf{Q}(\sqrt{D})$ в указанных двух случаях равен соответственно $4 D$ и $D$, а в качестве $\mathbf{Z}$-базисов могут быть взяты соответственно системы

$$
(1, \sqrt{D}) \text { и }\left(1, \frac{1+\sqrt{D}}{2}\right)
$$


Система чисел

$$
(1, w), \text { где } w=\frac{d+\sqrt{d}}{2},
$$

является $\mathbf{Z}$-базисом поля $\mathbf{Q}(\sqrt{D})$ в любом из указанных случаев.

Сигнатура поля $\mathbf{Q}(\sqrt{D})$ имеет вид $(0,1)$, если $D<0$, и $(2,0)$, если $D>0$. Поэтому согласно приведенной выше теореме Дирихле группа обратимых элементов кольца $\mathbf{Q}_{0}(\sqrt{D})$ является конечной циклической группой при $D<0$ и прямым произведением конечной циклической и бесконечной циклической групп при $D>0$. Точнее, она равна $<-1>$ при $D<-3$ и $<-1>x<\varepsilon>$ при $D>0$, где $\varepsilon>0-$ фундаментальная единица (которая легко находится по разложению числа $\sqrt{D}$ в периодическую цепную дробь). Число $\ln \varepsilon$ называется регулятором поля $\mathbf{Q}(\sqrt{D})$ и обозначается через $R(d)$, где $d-$ дискриминант поля (или просто буквой $R$ при известном $d$ ).

При изучении дробных идеалов обычно используется следующее стандартное представление дробного идеала $A \neq\{0\}:$

$$
A=q\left(a \mathbf{Z}+\frac{b+\sqrt{d}}{2} \mathbf{Z}\right),
$$

где

$$
q \in Q, a, b, c=\left(b^{2}-d\right) / 4 a \in Z, q>0, a>0, \text { НОД }(a, b, c)=1,-a<b \leq a .
$$

Кратко такой идеал обозначается выражением $q(a, b, c)$. Идеал $A$ называется примитивным, если $q=1$, и редуцированным, если

$$
\text { НОД }(a, b, c)=1,|b| \leq a \leq c \text { и } b \geq 0 \text {, если }|b|=a \text { или } a=c .
$$

Далее числа $a, b, c$ будут называться параметрами идеала $(a, b, c)$.

Так как каждый идеал, отличный от единичного идеала $\mathbf{K}_{0}$ и $(0)$, разлагается в произведение простых идеалов, то представляет особый интерес задача описания простых идеалов. Эта задача имеет следующее решение.

Множество простых идеалов кольца $\mathbf{Q}_{0}(\sqrt{D})$, содержащих нечетное простое число $p$, исчерпывается:

I а) при $\left(\frac{d}{p}\right)=1$ идеалами $P_{\mathrm{i}}=\left[p, b_{\mathrm{i}}+w\right], i=1,2$, где $\left(\frac{d}{p}\right)-$ символ Лежандра, a $b_{1}, b_{2}-$ различные решения сравнения по модулю $p$

$$
(2 x+d)^{2} \equiv d(\bmod p)
$$


I b) при $\left(\frac{d}{p}\right)=-1$ одним идеалом $(p)$;

I с) при $\left(\frac{d}{p}\right)=0$ одним идеалом $P=[p, w]$.

В случае $p=2$ множество простых идеалов кольца $\mathbf{Q}_{0}(\sqrt{D})$, содержащих число 2, исчерпывается:

II a) при $d \equiv 1(\bmod 8)$ идеалами $P_{1}=[2, w], P_{2}=[2,1+w]$;

II b) при $d \neq 1(\bmod 8), d \equiv 1(\bmod 4)$ одним идеалом $(2)$;

II c) при $d \equiv-1(\bmod 4)$ одним идеалом $P=[2,1+w]$;

II d) при $d \equiv 2(\bmod 4)$ одним идеалом $P=[2, w]$.

Заметим, что простое число $p$, порождающее простой идеал кольца $\mathbf{Z}$, не всегда порождает простой идеал в $\mathbf{Q}_{0}(\sqrt{D})$. А именно, в случаях I а) и II а) $(p)=P_{1} P_{2}$, в случаях I c), II c) и II d) $(p)=P^{2}$.

Идеалы $A, B$ кольца $\mathbf{Q}_{0}(\sqrt{D})$ называются эквивалентными, если существуют главные идеалы $(\alpha),(\beta)$ такие, что $(\alpha) A=(\beta) B$. Как и в общем случае, определяются дробные идеалы поля $\mathbf{Q}(\sqrt{D})$. Эквивалентность дробных идеалов $A, B$ означает существование числа $\alpha \in(\mathbf{Q}(\sqrt{D}))^{*}$, для которого $(\alpha) A=B$. Для порядка $h(d)$ группы классов идеалов имеют место формулы:

$$
h(d)=\frac{\sqrt{d}}{2 R(d)} L(1, \chi) \text { при } d>0, \quad h(d)=\frac{\sqrt{-d}}{\pi} L(1, \chi) \text { при } d<-4,
$$

в которых $\chi-$ примитивный характер по модулю $|d|$, a

$$
\mathrm{L}(1, \chi)=\prod_{p}\left(1-\frac{\chi(p)}{p}\right)^{-1},
$$

где произведение берется по всем простым числам $p \in \mathbf{Z}$. Используя свойства функции $\chi$, можно получить также конечные формулы:

$$
h(d)=-\frac{1}{R(d)} \sum_{\substack{0<x<d / 2 \\(x, d)=1}} \chi(x) \ln \sin \frac{\pi x}{d} \text { при } d>0,
$$




$$
h(d)=\frac{1}{d} \sum_{\substack{0<x<-d \\(x, d)=1}} \chi(x) x \text { при } d<0 .
$$

Очевидно, что приведенные формулы мало пригодны для нахождения точных значений величины $h(d)$ при больших значениях $d$, однако они используются для нахождения нижних и верхних оценок для $h(d)$.

Имеются и асимптотические оценки. Так, например, известно, что при $d \rightarrow \infty$

$$
(\sqrt{d})^{1-\varepsilon} \leq h(d) \leq(\sqrt{d})^{1+\varepsilon}, 0<\varepsilon<1
$$

Известно также среднее значение: $h(d)=c(\sqrt{d})$, где $c=0,461559 \ldots$ Исходя из этих и некоторых других теоретических результатов, а также учитывая многочисленные компьютерные эксперименты, принято считать, что, как правило, $h(d)$ есть величина порядка $\sqrt{d}$. Вместе с тем следует иметь в виду, что существуют квадратичные поля с малыми значениями величины $h(d)$. В частности, для колец главных идеалов $h(d)=1$.

Задача выяснения строения групп классов и вычисления их порядков является классической в теории алгебраических чисел. В последние годы внимание к ней значительно возросло в связи с применением группы классов в криптографии, поскольку в ряде алгоритмов дискретного логарифмирования в конечной группе существенную роль играет знание строения и порядка группы. Наилучшим по сложности алгоритмом нахождения величины $h(d)$ в настоящее время, по-видимому, можно считать алгоритм Маккерли (McCurley K.) [53, 32], который является некоторым улучшением алгоритма, предложенного в 1971 г. Шэнксом (Shanks D.) [68]. Алгоритм Шэнкса основан на трех главных идеях.

Первая идея - это использование формулы (2) для получения такого числа $B$, при котором выполнены неравенства

$$
B \leq h(d) \leq 2 B .
$$

Вторая идея - использование теоремы Лагранжа (порядок элемента в группе делит порядок группы) для распознавания принадлежности элемента подгруппе:

$$
f \in \mathrm{Cl}(d) \Rightarrow f^{h(d)}=1 .
$$

Третья идея - использование алгоритма «малый шаг-большой шаг» («babystep-giantstep») для ускорения нахождения интервала $[B, 2 B]$. 
Маккерли заменил проверку соотношений вида (3) проверкой случайно набранных соотношений между образующими группы $\mathrm{Cl}(d)$ с помощью достаточно простого алгоритма Шуфа (Schoof R. J.) [66] для построения системы образующих группы $\mathrm{Cl}(d)$. В итоге для сложности алгоритма вычисления числа $h(d)$ при выполнении обобщенной гипотезы Римана получена субэкспоненциальная оценка $L_{d}\left(2^{1 / 2}\right)$. В [53] отмечается также, что, используя метод Видемана для вычисления определителей, встречающихся в алгоритме разреженных матриц, можно снизить полученную оценку до $L_{d}\left(3 \cdot 2^{1 / 2} / 4\right)$. Заметим, что $3 \cdot 2^{1 / 2} / 4 \approx 1,06$.

Отметим в заключение данного пункта, что при изучении идеалов и группы классов идеалов поля $\mathbf{Q}(\sqrt{D})$ обычно используют связь между идеалами и квадратичными формами от двух переменных над $\mathbf{Z}$.

Для любой такой формы

$$
f(x, y)=a x^{2}+b x y+c y^{2}
$$

числа $d(f)=b^{2}-4 a c$ и $S(f)=$ НОД $(a, b, c)$ называются соответственно ее дискриминантом и содержанием. Форма $\mathrm{f}$ называется примитивной, если $S(f)=1$. Если при этом $d<0$ и $a>0$, то форма $f$ положительно определена. Множество всех примитивных квадратичных форм с дискриминантом $d$ обозначим через $F(d)$. Обычным образом вводится эквивалентность квадратичных форм относительно специальной линейной группы $\operatorname{SL}(2, \mathbf{Z})$ (т. е. группы $2 \times 2$-матриц над $\mathbf{Z}$ с определителем 1$)$. При этом величины $d(f)$ и $S(f)$ являются инвариантами классов эквивалентных форм. В итоге все примитивные формы из $F(d)$ разбиваются на классы эквивалентных форм. Одна из главных задач теории квадратичных форм заключается в отыскании алгоритма распознавания эквивалентности примитивных форм с одинаковым дискриминантом $d$. Сравнительно просто решается эта задача при $d<0$. Эта задача сводится к положительно определенным формам, т. е. к формам вида (4) при $a>0$. Для них, как и для идеалов, вводится редуцированная квадратичная форма и доказывается, что в любом классе эквивалентных положительно определенных квадратичных форм существует единственная редуцированная форма. Тем самым задача сведена к построению алгоритма редуцирования форм, т. е. преобразования заданной формы в редуцированную с помощью матриц из $\mathrm{SL}(2, \mathbf{Z})$.

Взаимно однозначное соответствие между положительно определенными квадратичными формами дискриминанта $d<0$ и идеалами кольца $\mathbf{Q}_{0}(\sqrt{D})$ с дискриминантом $d$ осуществляется сопоставлением форме вида (4) примитивного идеала

$$
(a, b, c)=a \mathbf{Z}+\frac{-b+\sqrt{d}}{2} \mathbf{Z} .
$$


При этом эквивалентным формам соответствуют эквивалентные идеалы, и обратно: редуцированным формам соответствуют редуцированные идеалы.

\section{3. О криптосистемах с открытым ключом, основанных на группах классов мнимых квадратичных полей}

Идея использования групп классов квадратичных полей для построения схем с открытым ключом впервые была высказана Маккерли в 1988 г. [52]. Главная мотивация этой идеи заключалась в расширении списка используемых в криптографии трудных задач теории чисел. В связи с его предложением к используемым задачам факторизации целых чисел и дискретного логарифмирования в конечных полях добавились известные своей сложностью задачи вычисления группы классов квадратичных полей и дискретного логарифмирования в таких группах.

В том же году эту идею частично реализовали Бухман и Вильямс (Buchmann J., Williams H.) [29], представив криптосистему типа ДиффиХеллмана с помощью группы классов мнимого квадратичного поля. Для построения своей схемы они использовали редуцированные идеалы поля $\mathbf{Q}_{0}(\sqrt{D})$ с дискриминантом $d<0$. Такие идеалы оказались полезными в связи с тем, что каждый класс эквивалентных идеалов содержит единственный редуцированный идеал, и потому умножение классов сводится к умножению редуцированных представителей этих классов с последующим редуцированием результата. Кроме того, использование редуцированных идеалов облегчает операцию умножения идеалов, поскольку параметры таких идеалов ограничены числом $\sqrt{d}$.

Известно, что если идеал $A$ редуцированный, то $a<\sqrt{-d / 3}$, а с другой стороны, если $a<\sqrt{-d / 4}$, то идеал $A-$ редуцированный.

Опишем теперь криптосистему, предложенную в [29]. Эта система служит для выработки общего ключа абонентами связи U, V и выглядит следующим образом. Абоненты U и V согласованно и открыто выбирают свободное от квадратов и отличное от 0 и 1 целое число $D<0$ и редуцированный идеал $I$ кольца $\mathbf{Q}_{0}(\sqrt{D})$. Далее все происходит по классической схеме Диффи-Хеллмана: U и V выбирают случайно соответственно целые числа $x$ и $y$ из промежутка $(1,|\sqrt{D}|)$, затем $\mathrm{U}$ высылает открыто $\mathrm{V}$ идеал $I^{x}$, а $\mathrm{V}$ высылает $\mathrm{U}$ идеал $I^{y}$. Теперь $\mathrm{U}$ и $\mathrm{V}$ возводят идеалы $I^{y}$ и $I^{x}$ соответственно в степени $x$ и $y$. При этом во избежание появления слишком больших чисел абоненты U, V после каждого применения операции умно- 
жения применяют к результату алгоритм редуцирования. В итоге каждый из абонентов $\mathrm{U}, \mathrm{V}$ получит редуцированный идеал $J$, эквивалентный идеалу $I^{x y}$. Так как каждый класс эквивалентных идеалов содержит единственный редуцированный идеал, то полученные идеалы будут совпадать. Идеал $J$ и определяет общий ключ. В [29] в качестве общего ключа предлагается выбирать норму идеала $J$. Таким образом, секретным ключом является пара чисел $x, y$, а открытым - идеалы $I, I^{x}, I^{y}$.

Из приведенного описания схемы видно, что сложность получения общего ключа определяется сложностью алгоритма редуцирования идеалов и умножения редуцированных идеалов. Ниже приводятся соответствующие алгоритмы.

\section{Алгоритм умножения идеалов}

Если $A_{i}=\left(a_{i}, b_{i}, c_{i}\right), i=1,2$, два идеала, то $A_{1} A_{2}=A_{3}=d_{0}\left(a_{3}, b_{3}, c_{3}\right)$, где

$$
\begin{gathered}
a_{3}=d_{1} \frac{a_{1} a_{2}}{d_{0}^{2}}, b_{3}=b_{2}-b_{3}=b_{2}-\frac{2 a_{2}}{d_{0}}\left(v\left(s+b_{2}\right)-w c_{2}\right), \\
c_{3}=\frac{b_{3}^{2}-d}{4 a}, s=-\frac{b_{1}+b_{2}}{2}, \\
d_{0}=\text { НОД }\left(a_{1}, a_{2}, s\right), u, v, w \in \mathbf{Z}, u a_{1}+v a_{2}+w s=d_{0}, \\
d_{1}=\text { НОД }\left(a_{1}, a_{2}, s, c_{1}, c_{2}, b_{1}-s\right) .
\end{gathered}
$$

Заметим, что если хотя бы один из идеалов $A_{1}, A_{2}$ примитивен, то $d_{1}=1$.

Из приведенного результата видно, что сложность умножения редуцированных идеалов не превосходит $O\left(\log ^{2} d\right)$.

Алгоритм редуцирования идеалов является наиболее сложной частью в реализации всей схемы выработки ключа. Поэтому редуцированию идеалов посвящено большое число работ разных авторов. Классические алгоритмы редуцирования бинарных квадратичных форм над $\mathbf{Z}$ восходят к Л. Лагранжу и К. Гауссу. В последние годы в связи с криптографическими приложениями появился цикл работ, в которых представлен ряд новых алгоритмов и модификаций классических алгоритмов редуцирования с оценками их сложности (см. $[6,15,49,60,65,67,68])$. Обзор по методам редуцирования содержится в [49]. Здесь мы опишем наиболее простую по сложности и по описанию схему алгоритма редуцирования, предложенного в работе [15]. Ее описание ведется на языке квадратичных форм.

Квадратичную форму (4) обозначим для краткости в виде $f=[a, b, c]$. Примитивные и редуцированные формы определяются теми же условиями на $a, b, c$, что и для соответствующих им идеалов. В нашем случае, когда $d<0$, форма $f$ является положительно определенной при $a>0$ и отрицательно определенной при $a<0$. Ясно, что достаточно рассмотреть лишь 
один из этих случаев. Для определенности будем считать, что $a>0$, и множество всех примитивных положительно определенных форм с дискриминантом $d$ обозначим через $F^{+}(d)$. Введем еще следующие обозначения:

$$
M_{u}=\left(\begin{array}{cc}
0 & 1 \\
-1 & u
\end{array}\right), \quad t_{u}(f(x, y))=f\left((x, y) M_{u}\right) .
$$

Легко проверить, что если $f=[a, b, c] \in F^{+}(d)$, то

$$
t_{u}(f)=\left[a_{1}, b_{1}, c_{1}\right] \in F^{+}(d) \text { и } a_{1}=c, \quad b_{1}=-b+2 c u, \quad c_{1}=a-b u+c u^{2} .
$$

Используя такие преобразования, опишем сначала классический алгоритм редуцирования нередуцированной формы $f \in F^{+}(d)$ (см., например, [15]).

Подействуем на форму $f$ преобразованием $t_{u}$, выбрав $u$ из условия

$$
-a_{1}<2 a u+b<a_{1} .
$$

Получим форму (5). Если при этом $c_{1}>a_{1}$, то она редуцирована. Если $c_{1}=a_{1}$ и $b_{1}<0$, то, применив к ней преобразование $t_{0}$, получим редуцированную форму $f_{1}=\left[c_{1},-b_{1}, a_{1}\right]$. Остается случай $c_{1}<a_{1}$. В этом случае применим к $f_{1}$ ту же процедуру, которую выше применили к $f$.

Продолжая этот процесс, мы получим последовательность форм $\left[a_{i}, b_{i}, c_{i}\right]$, где $a<a_{1}<a_{2}<\ldots$, и на некотором шаге с номером $k-$ редуцированную форму $f_{k}=\left[a_{k}, b_{k}, c_{k}\right]$.

Оценим число шагов $k$. Можно доказать, что если $a \leq \sqrt{d}$, то либо $f_{1}$ редуцирована, либо из нее получается редуцированная форма за одно преобразование. Значит, в этом случае $k<3$. Если $a>\sqrt{d}$, то $a_{1}<a / 2$. Учитывая, что при $a<\sqrt{-d / 4}$ форма $f_{1}$ редуцирована, получаем:

$$
k \leq\left[\log _{2} \frac{a}{\sqrt{-d}}\right]+2 .
$$

Отсюда выводится, что сложность редуцирования формы $f=[a, b, c]$ не превосходит $O\left(\|f\|^{3}\right)$ двоичных операций, где $\|f\|-$ длина двоичной записи числа $|a b c|$. При этом предполагается, что бинарная сложность вычисления $a+b, a b$ равна соответственно $O(\max \{\|a\|,\|b\|\}), O(\|a\| \cdot\|b\|)$.

В работе [15] приводится и новая модификация описанного алгоритма. С этой целью ее авторы вводят величину

$$
v(f)=\operatorname{sign}(c)\left[\frac{|c|+b}{2|c|}\right]
$$


и доказывают, что если формы $f, t_{v(f)}(f)$ и $\left(t_{v(f)}\right)^{2}(f)$ не редуцированы и выполняется условие $-|a|<b \leq|a|$, то $|v(f)| \geq 2$ и $|a| \geq|v(f)||c|$. Пользуясь этим утверждением, они уточняют оценку сложности алгоритма редуцирования до $O\left(\|f\|^{2}\right)$. Такую же оценку они получают и при $d>0$.

Вернемся к схеме, предложенной в [29]. Задача нахождения общего ключа в этой схеме сводится к проблеме дискретного логарифмирования (ПДЛ) в конечной абелевой группе $\mathrm{Cl}(d)$, которая существенно зависит от порядка и строения группы $\mathrm{Cl}(d)$. Авторами было доказано, что в общем случае ПДЛ в группе $\mathrm{Cl}(d)$ не проще проблемы факторизации целых чисел.

Указанная работа Бухмана и Вильямса инспирировала большой цикл работ тех же и многих других авторов по обобщениям и модификациям предложенной схемы, по оценкам сложности реализации процессов шифрования и расшифрования в таких схемах и по криптографическому анализу таких схем, сводящемуся главным образом к ПДЛ в группе $\mathrm{Cl}(d)$.

Один из методов логарифмирования в группе $\mathrm{Cl}(d)$ при $d<0$, применявшийся ранее к решению ПДЛ в других группах и называемый индексметодом, использовал в 1989 г. Маккерли (см. [53], [32]). Рассмотренный в этих работах алгоритм логарифмирования в группе $\mathrm{Cl}(d)$ основан на знании порядка $h(d)$ этой группы, который находился указанным в п. 2 методом из [53], являющимся улучшенной модификацией метода Шэнкса [68].

Далее индекс-метод был развит и упрощен в [30], [22]. Его идея проста и основана на представлении группы в виде прямого произведения циклических групп.

Пусть

$$
\mathrm{Cl}(d)=\left\langle g_{1}>\times \ldots \times<g_{r}>, \quad \text { ord }\left(g_{i}\right)=n_{i}, i=1, \ldots, r,\right.
$$

и требуется в группе $\mathrm{Cl}(d)$ решить относительно $x$ уравнение

$$
\alpha^{x}=\beta, \quad \alpha, \beta \in \mathrm{Cl}(d) .
$$

Для этого при наличии информации (6) достаточно представить $\alpha, \beta$ виде

$$
\alpha=\prod_{i=1}^{r} g_{i}^{a(i)}, \quad \beta=\prod_{i=1}^{r} g_{i}^{b(i)}
$$

и решить систему линейных сравнений

$$
a(i) x \equiv b(i)\left(\bmod n_{i}\right), i=1, \ldots, r .
$$

Наименьшее положительное решение сравнения $a(i) x \equiv b(i)\left(\bmod n_{i}\right)$ называют логарифмом элемента $g_{i}^{b(i)}$ при основанию $g_{i}^{a(i)}$ по модулю $n_{i}$. В теории чисел вместо слова «логарифм» используется слово «индекс». Отсюда и ведет свое название индекс-метод. 
Главная трудность индекс-метода заключается в нахождении представлений (6) и (7). Для их нахождения используется основная теорема теории идеалов о том, что любой идеал кольца $\mathbf{Q}_{0}(\sqrt{D})$, отличный от (0) и (1), представляется в виде произведения простых идеалов. Поэтому предлагаемый алгоритм начинается с выбора некоторой системы простых идеалов $P_{1}, \ldots, P_{k}$ и поиска соотношений вида

$$
\prod P_{i}^{t(i)}=(1),
$$

где произведение берется по $i=1, \ldots, k$. Затем находится базис $\left(b_{1}, \ldots, b_{k}\right)$ целочисленной решетки, составленной из найденных наборов показателей $(t(1), \ldots, t(k))$, и для матрицы $M$, строками которой являются наборы $b_{1}, \ldots, b_{k}$, находится каноническая форма

$$
S=U M V=\operatorname{diag}\left(n_{1}, \ldots, n_{r}, 1, \ldots, 1\right) .
$$

Если $U=(u(i j))$ и $Q_{i}=\prod P_{j}^{u(j i)}, j=1, \ldots r$, то имеет место разложение (6), в котором $g_{i}$ есть класс идеалов с представителем $Q_{i}$. Обратно, если $V^{-1}=(v(i j))$, то $P_{i}=\prod Q_{j}^{v(i j)}$. Таким образом, если будет найдено выражение элемента из $\mathrm{Cl}(d)$ через классы $h_{1}, \ldots, h_{k}$ с представителями $P_{1}, \ldots, P_{k}$, то по нему легко будет найти и его представление через классы $g_{1}, \ldots, g_{r}$. Поэтому для нахождения представлений (7) находятся сначала представления элементов $\alpha, \beta$ через $h_{1}, \ldots, h_{k}$.

Для этого выбирается случайно набор чисел $(v(1), \ldots, v(k)), 0 \leq v(i) \leq d-1$, и вычисляется редуцированный идеал $B$, эквивалентный идеалу $A \prod P_{i}^{v(i)}$, где $A-$ редуцированный идеал класса $\alpha$. Этот процесс продолжается до тех пор, пока не получится идеал $B$, представимый в виде $\prod P_{i}^{w(i)}$. Тогда $\alpha=\prod h_{i}^{w(i)-v(i)}$. Таким же способом находится аналогичное представление и для $\beta$. Теперь от полученных представлений легко перейти к представлениям типа (7).

Доказывается, что если в качестве $P_{1}, \ldots, P_{k}$ использовать все простые идеалы с нормой, не превосходящей величины $L_{d d}(\delta)$, то нужный идеал $B$ получим с вероятностью $L_{d \mid}(-1 / 4 \delta)$. При этом сложность алгоритма будет оцениваться величиной $L_{d l}(c)$.

В [22] этот алгоритм реализован в виде компьютерной программы и найдены параметры $k, r, n_{i}, h(d)$ для $d=-\left(4 \cdot 10^{t}+4\right)$ при $t=24,29,34$, а также для $d=-4 F_{7}$, где $F_{7}-7$-е число Ферма. Эксперименты подтвердили по- 
лученные оценки сложности. При этом параметры $k$ и $r$ принимали соответственно следующие значения: $k=1404,1902,2609,3257$ и $r=3,3,4,2$.

Некоторое уточнение оценок сложности реализации процесса выработки общего ключа и ПДЛ в группе $\mathrm{Cl}(d)$ было получено в работах $[24,70,45]$ и др. В частности, в [24] было доказано, что сложность реализации имеет порядок $O\left(\log ^{4}(-d)\right)$. В работе [38] был дан обзор различных алгоритмов решения ПДЛ. Из него следовало, что алгоритм решения проблемы ПДЛ индекс-методом при выполнении обобщенной гипотезы Римана имеет сложность $L_{|d|}\left(3 \cdot 2^{1 / 2} / 4\right)$, и применение таких методов как $\lambda$-метод Полларда и метод Полига-Хеллмана не привело к улучшению оценки сложности, полученной по индекс-методу. В [46] к решению ПДЛ был применен метод многополиномиального квадратичного решета, который на практике имел сложность $\mathrm{L}_{d \mid}(1)$ хотя доказательства этой оценки не было. На основании сделанного обзора авторы делают вывод о том, что на тот период ПДЛ в группе $\mathrm{Cl}(d)$ имела оценку сложности бо́льшую, чем проблема факторизации целых чисел и ПДЛ в мультипликативной группе конечного поля. Следовательно, группа классов мнимого квадратичного поля является потенциальной альтернативой другим группам, используемым при построении криптографических протоколов.

В указанной обзорной работе авторы приводят также определенные соображения по выбору дискриминанта $d$ поля $\mathbf{Q}(\sqrt{D})$. Суть их сводится к тому, что для обеспечения стойкости схемы к индексному и другим методам $d$ нужно выбирать так, чтобы число $h(d)$ было достаточно большим и не гладким, т. е. имело большой простой делитель. Первое требование реально можно выполнить с помощью нахождения нижних оценок величины $h(d)$ из формулы (2). Однако эффективных методов выбора дискриминанта $d$, обеспечивающих выполнение второго требования к $h(d)$, пока не найдено. Имеются лишь экспериментальные наблюдения, свидетельствующие о том, что чаще всего это требование выполняется.

\section{4. О криптосистемах с открытым ключом, основанных на группах классов идеалов немаксимальных порядков мнимых квадратичных полей}

Из всего сказанного в п. 3 видно, что с точки зрения практического применения в криптографии группа $\mathrm{Cl}(d)$ мнимого квадратичного поля $\mathbf{Q}(\sqrt{D})$ имеет свои достоинства и недостатки. Главным ее достоинством является большая сложность (на сегодняшний день) решения ПДЛ, связанная, в основном, со сложностью вычисления группы $\mathrm{Cl}(d)$ и ее порядка $h(d)$. В то же время сложность последней задачи является ее недостатком, по- 
скольку затрудняет выбор дискриминанта $d$, обеспечивающего требования по стойкости, а также делает невозможным ее использование для построения криптосхем типа RSA, Эль-Гамаля и других схем, в которых необходимо знание порядка или хотя бы экспоненты используемой группы. Кроме того, при использовании группы $\mathrm{Cl}(d)$ увеличивается сложность реализации схемы открытого распределения ключей по сравнению со схемами, основанными на группах конечных полей или эллиптических кривых.

Для преодоления этих недостатков в работе [41] было предложено использовать группу классов (идеалов) немаксимального порядка мнимого квадратичного поля. Эта работа нашла достаточно широкий отклик и инициировала серию статей с предложениями использовать эту идею для построения различных криптографических систем с открытым ключом, а также по анализу таких систем (см. [19,37-43,45,57,58]).

Приведем нужные нам факты о порядках квадратичных полей. Порядком квадратичного поля $\mathbf{Q}(\sqrt{D})$ называют всякое подкольцо кольца $\mathbf{Q}_{0}(\sqrt{D})$, являющееся $\mathbf{Z}$-модулем ранга $r \leq 2$. В интересующем нас случае, когда $D<0$, любой порядок поля можно представить как множество чисел вида

$$
q\left(a+\frac{b\left(d_{1}+\sqrt{d_{1}}\right)}{2}\right),
$$

где $d_{1} \in \mathbf{Z}, d_{1}<0, d_{1} \equiv 0$ или $1(\bmod 4)$ и $d_{1}$ не является полным квадратом в $\mathbf{Z}$, $a, b \in \mathbf{Z}, a>0,-a<b \leq a, q \in \mathbf{Q}$. Если при этом $d_{1}$ свободно от квадратов при $d_{1} \equiv 1(\bmod 4)$ и $d_{1} / 4$ свободно от квадратов при $d_{1} \equiv 0(\bmod 4)$, то порядок $O\left(d_{1}\right)$ называется максимальным, в противном случае он называется немаксимальным. Следовательно, максимальный порядок $O\left(d_{1}\right)$ есть кольцо $\mathbf{Q}_{0}(\sqrt{D})$ поля $\mathbf{Q}(\sqrt{D})$ с дискриминантом $d_{1}$. Если же порядок $O\left(d_{1}\right)$ не максимальный, то $d_{1}$ представимо в виде $d_{1}=f^{2} d$, где $f, d \in \mathbf{Z}$, а $d$ свободно от квадратов и потому является дискриминантом максимального порядка. Заметим, что при этом, очевидно, $\mathbf{Q}\left(\sqrt{d_{1}}\right)=\mathbf{Q}(\sqrt{d})$, и потому $O\left(d_{1}\right) \subset O(d)$, т. е. немаксимальный порядок $\mathbf{Q}\left(\sqrt{d_{1}}\right)$ входит в кольцо $\mathbf{Q}_{0}(\sqrt{D})$ с дискриминантом $d$.

Для немаксимального порядка $O\left(d_{1}\right)$ точно так же, как и для кольца $\mathbf{Q}_{0}(\sqrt{D})$, определяются понятия идеала, главного идеала, редуцированного идеала, дробного идеала, эквивалентности дробных идеалов, группы классов идеалов $\mathrm{Cl}\left(d_{1}\right)$ и ее порядка $h\left(d_{1}\right)$. Доказывается, что в каждом классе группы 
$\mathrm{Cl}\left(d_{1}\right)$ существует единственный редуцированный идеал, и, значит, операция умножения классов группы $\mathrm{Cl}\left(d_{1}\right)$ сводится к редуцированию идеалов и умножению редуцированных идеалов.

В работе [41] было замечено, что в случае, когда $d_{1}=f^{2} d<0$, существует достаточно простая связь между числами $h\left(d_{1}\right)$ и $h(d)$, позволяющая легко вычислять $h\left(d_{1}\right)$ по $h(d)$ и $f$ :

$$
h\left(d_{1}\right)=h\left(f^{2} d\right)=h(d) f \prod\left(1-\left(\frac{d}{q}\right)\right),
$$

где $\left(\frac{d}{q}\right)$ - символ Якоби, а произведение берется по всем простым делителям $q$ числа $f$. Поэтому немаксимальные порядки с дискриминантами вида $d_{1}=f^{2} d<0$ и с легко вычислимой величиной $h(d)$, в частности, для тотально немаксимальных порядков, т. е. при $h(d)=1$, могут использоваться для построения схем типа Рабина, RSA и Эль-Гамаля.

Схема типа Эль-Гамаля, основанная на мнимых порядках с дискриминантами вида $d_{1}=f^{2} d<0$ была представлена в [41]. Вскрытие такой схемы влекло факторизацию ее дискриминанта $d_{1}$. Однако известная в то время оценка сложности реализации таких схем существенно превосходила сложность реализации существующих схем, и потому они представляли в основном лишь теоретический интерес.

Вскоре Паулюс и Такаги (S. Paulus, T. Takagi) предложили эффективную реализацию криптосхем с открытым ключом, основанных на немаксимальных квадратичных порядках с отрицательным дискриминантом $d_{1}$ (опубликовано позднее, в 1998 г. [59]). Сложность процесса расшифрования в их схемной реализации была на тот период наименьшей по сравнению с существующими аналогами и оценивалась величиной $O\left(\log ^{2} d_{1}\right)$. Главная идея упрощения реализации заключалась в использовании введенного еще в работе [41] изоморфизма, переводящего процесс реализации от немаксимального порядка к соответствующему максимальному порядку. Этот результат существенно повысил интерес к схемам, основанным на немаксимальных порядках. В 1998-1999 гг. были представлены основанные на этом принципе схемы, аналогичные схемам Рабина и RSA [43], а также схема цифровой подписи типа DSA [19]. При этом для аналога схемы Рабина (основанной на извлечении квадратного корня в кольце $\mathbf{Z} / m \mathbf{Z}$ при составном $m$ ) использовались дискриминанты вида $d_{1}=f^{2} d<0$, где $f=q, d=-p$, если $p \equiv 3$ $(\bmod 4)$, и $d=-4 p$ в остальных случаях, $p, q-$ простые числа. Для схемы типа RSA использовались дискриминанты вида $d_{1}=f^{2} d<0$, где $f=p q-$ 
произведение двух простых чисел $p, q$, а $d$ таково, что $h(d)=1$. Доказано, что в последнем случае

$$
h\left(d_{1}\right)=\left(p-\left(\frac{d_{1}}{p}\right)\right)\left(q-\left(\frac{d_{1}}{q}\right)\right) .
$$

Вскрытие такой схемы имеет такую же сложность, что и факторизация числа $f=p q$. Поэтому по стойкости такой аналог RSA не уступает оригинальной схеме RSA. Однако представленные схемы типа RSA обладают тем преимуществом, что способны противостоять атакам при нескольких экспонентах шифрования и атакам на специально подобранный шифртекст.

Новая, еще более простая, арифметика в немаксимальных порядках была предложена в [40], где неэффективные операции умножения и редуцирования идеалов заменены простыми манипуляциями с образующими элементами группы. Эта техника использовалась также для аналога схемы цифровой подписи, в котором время порождения подписи было сравнимо со стандартом DSA в конечном поле. Авторы указывали также, что остаются широкие возможности для дальнейшего развития рассматриваемых схем.

Стойкость таких схем, как и для максимальных порядков, зависит от выбора дискриминанта $d_{1}$. В связи с этим представляет интерес результат работы [45], где было доказано, что проблема дискретного логарифмирования в группе классов тотально немаксимального порядка с дискриминантом $d_{1}=p^{2} d$ при простом $p$ сводится со сложностью $O\left(\log ^{3} p\right)$ к аналогичной проблеме в мультипликативной группе поля $\operatorname{GF}(p)$ при $\left(\frac{d}{p}\right)=1$ и поля $\operatorname{GF}\left(p^{2}\right)$ при $\left(\frac{d}{p}\right)=-1$.

В работе [37] обсуждаются вопросы практического применения таких систем, которые авторы назвали NICE-системами (New Ideal Coset Encryption). Отмечается, что в них существенно проще и быстрее осуществляется процесс расшифрования, чем в известных криптосистемах. В частности, приводится сравнительная таблица с классической системой RSA. Приведем один пример из этой таблицы.

При $d_{1}=f^{2} d \approx 1536$ и примерно такой же величине модуля $n=p q$ схемы RSA время на дешифрование в схеме RSA равно 751,3 ms, а в NICE-системе $6,2 \mathrm{~ms}$. В связи с этим авторы считают, что NICE-системы являются вполне подходящими для использования в смарт-картах, а также в такой ситуации, когда на центральном пункте связи требуется быстро расшифровывать много сообщений. 
В заключение данного параграфа укажем еще работу [38], в которой приведен обзор результатов по криптосистемам, основанным на использовании мнимых квадратичных полей. В ней обсуждаются история развития, современное (на 2000 г.) состояние и дальнейшие направления исследований по таким системам.

\section{5. О криптосистемах с открытым ключом, основанных на классах идеалов вещественных квадратичных полей}

В 1989 г. почти одновременно с работой [29] ее авторы Д. Бухман и X. Вильямс вместе с Р. Шейдлером (R Scheidler) опубликовали статью [27], в которой предложили схему открытого распределения ключей с использованием дробных идеалов вещественного квадратичного поля $\mathbf{Q}(\sqrt{D}), D>0$. В этом случае проблема построения схемы решается значительно труднее по сравнению со схемами, основанными на использовании мнимого квадратичного поля. Дело в том, что при $D>0$ каждый класс дробных идеалов содержит много редуцированных идеалов, и потому при редуцировании одного и того же идеала разные лица могут получить разные идеалы. Поэтому здесь авторы ограничились рассмотрением лишь главных идеалов. Все такие идеалы образуют один класс эквивалентности. Известно, что в нем, как и в любом другом классе идеалов, существует лишь конечное множество редуцированных идеалов и известен алгоритм построения последовательности всех редуцированных идеалов класса по любому из них с использованием периодических цепных дробей [21]. Множество всех редуцированных главных идеалов обозначим буквой $S$.

Главная идея построения схемы открытого распределения ключей заключается в использовании введенных ранее в [69] понятий инфраструктуры вещественного квадратичного поля, расстояний $\delta$ между идеалами из $S$ и между идеалами из $S$ и действительными числами. В общем плане протокол выработки общего ключа абонентами А и В выглядит следующим образом.

Абоненты А и В открыто выбирают небольшое действительное число $c>0$, идеал $I(c)$, ближайший к числу $c$, и расстояние $\delta(\mathrm{I}(c), c)$.

Далее А выбирает случайно число а из промежутка $(1, \sqrt{D})$, вычисляет $I(a c)$ и $\delta(I(a c), a c)$ и отправляет полученные результаты В. Аналогично В выбирает $b$, вычисляет $I(b c)$ и $\delta(I(b c), b c)$ и отправляет их А.

Теперь по имеющимся данным каждый из абонентов А, В вычисляет идеал $I(a b c)$, который и определяет общий ключ.

Следует отметить, что в действительности вычисляются не точные значения идеалов и их расстояний до идеала $I$ или действительного числа $x$, 
а некоторые их приближенные значения, но точность приближений рассчитывается так, чтобы конечные результаты были одинаковыми.

Анализу сложности процессов зашифрования, расшифрования и восстановления ключа в указанных криптосистемах посвящено (полностью или частично) большое число работ из приведенного ниже списка [9-70]. Одним из недостатков таких систем является сложность нахождения идеала, ближайшего к заданному числу. В работе [62] указывается способ построения двух ближайших соседних к заданному идеалу главных редуцированных идеалов и предлагается каждому из партнеров связи иметь по два идеала для порождения общего ключа. Кроме того, указывается оценка для числа точных значащих символов в приближенных значениях порождающих идеалов. Эта оценка улучшена в [48]. В [44] предложен путь, позволяющий избежать неоднозначности в выборе ближайшего идеала с помощью обмена дополнительными импульсами. В [62] доказано, что проблема стойкости системы сводится к проблеме главного идеала, которая формулируется следующим образом. По заданному идеалу $(a, b, c)$ выяснить, является ли он главным, и если да, то найти порождающий его элемент. Известно, что эта проблема не проще проблемы факторизации целых чисел. В [70] предложена определенная модификация индекс-метода, которая при выполнении обобщенной гипотезы Римана решает проблему дискретного логарифмирования (ПДЛ) в $S$ с субэкспоненциальной сложностью $L_{D}(\sqrt{2})$. Доказано также, что наличие полиномиального алгоритма для ПДЛ в $S$, т. е. по существу для решения проблемы расстояния между идеалами из $S$ и действительными числами, влечет наличие полиномиальных алгоритмов для вскрытия ключа и для факторизации числа $D$. В $[46,50]$ приводятся экспериментальные оценки алгоритма, основанного на использовании некоторой разновидности квадратичного решета. Эксперименты показывают, что этот алгоритм более эффективен, однако асимптотические оценки не улучшает.

В работе [24] проведен подробный анализ всех алгоритмов, необходимых для практической реализации предложенной схемы. Указаны асимптотические оценки сложности алгоритмов. Наиболее сложным из них является алгоритм нахождения приближенного значения для $I(x y)$ и $\delta(I(x y), x y)$ по заданным приближенным значениям соответствующих величин для $x$ и числу $y>0$. Сложность этого алгоритма составляет $O\left(\log ^{2} D\right)$ арифметических операций над числами порядка $O(\log D)$.

Представленная в [27] схема была обобщена и развита в работе [14], опубликованной в сборнике Crypto-94. В этой работе для построения схем с открытым ключом использовались не только кольца целых алгебраических чисел вещественных квадратичных полей, но и произвольные порядки этих 
полей. Ниже мы приведем краткое описание результатов указанной работы для квадратичных полей.

В ней порядок $O_{D}$ с дискриминантом $D>0$ представляется в виде

$$
O_{D}=\mathbf{Z}+\mathbf{Z} \frac{D+\sqrt{D}}{2} .
$$

Поле частных порядка $O_{D}$ совпадает с $\mathbf{Q}(\sqrt{D})$. Любой дробный идеал $A$ порядка $O_{D}$ можно представить в виде

$$
A=q(\mathbf{Z} a+\mathbf{Z}(b+\sqrt{D}) / 2 a), \text { где } a, b \in \mathbf{Z}, q \in \mathbf{Q}, a>0, q>0, c=b^{2}-D / 4 a \in \mathbf{Z} .
$$

При этом числа $a, q$ определяются однозначно, а число $b-$ однозначно по модулю $2 a$. Чтобы такое представление было полностью однозначным, достаточно условиться выбирать $b$ так, что

$$
-2 a+\sqrt{D}<b<\sqrt{D} \text { при } a<\sqrt{D} \quad \text { и } \quad-a<b<a \text { при } a>\sqrt{D} .
$$

В работе рассматриваются именно такие идеалы и только при $q=1$. Тогда идеал $A$ можно записать в виде $A=\mathbf{Z} a+\mathbf{Z} \gamma(A)$, где $\gamma(A)=(b+\sqrt{D}) / 2 a$. Идеал $A$ называется редуцированным, если для числа $\gamma(A)$ и сопряженного с ним числа $\gamma(A)^{\prime}$ выполняются условия $\chi(A)>1,0<\gamma(A)^{\prime}<1$. Если идеал $A$ редуцированный, то $a<\sqrt{D}$ и $|b|<\sqrt{D}$. Отсюда следует, в частности, конечность множества редуцированных идеалов порядка $O_{D}$. Множество всех главных редуцированных идеалов порядка $O_{D}$ обозначим через $S_{D}$.

Если идеалы $A, B$ эквивалентны и $A=\alpha B$, то число $\alpha \in \mathbf{Q}(\sqrt{D})^{*}$ определяется не однозначно, а именно, его можно заменить числом $\beta=\alpha \eta$, где $\eta$ - любая единица порядка $O_{D}$, и потому $\eta= \pm \varepsilon^{k}$, где $\varepsilon-$ фундаментальная единица порядка $O_{D}, k \in \mathbf{Z}$. Отсюда следует, что

$$
\log |\beta|=\log |\alpha|+k R_{D},
$$

где $R_{D}=\log \varepsilon-$ регулятор порядка $O_{D}$. При этом класс вычетов $\log |\alpha|+R_{D} \mathbf{Z}$ называется расстоянием между указанными идеалами $A, B$ и обозначается через $\delta(A, B)$ (и через $\delta(A)$, если $B=O_{D}$ ).

Теперь любому действительному числу $k$ сопоставляется пара $(A, c)$, где $A-$ ближайший к числу $k$ главный редуцированный идеал, а $c-$ расстояние от $A$ до $k$. При этом $A$ и $k$ определяются следующим образом:

$$
\begin{gathered}
A=(1 / \alpha), c=\log |\alpha|-k, \text { где } \alpha \text { выбирается так, } \\
\text { что }|\log | \alpha|-k|<|\log | \beta|-k| \text { для всех } \beta \in \mathrm{O}_{\mathrm{D}} .
\end{gathered}
$$


Если этими условиями $A$ определяется не однозначно, то требуют еще, чтобы было $c>0$.

Далее определяется понятие степени пары $(A, c)$. Пусть $m$ - натуральное число. Тогда $m$-й степенью пары $(A, c)$ называется пара $(B, r)$, в которой $B$ есть редуцированный главный идеал, ближайший к числу $x=m(\delta(A)+c)$, a $r=\delta(x, B)$. Это определение корректно, т. е. результат не зависит от выбоpa $\delta$. Полученная пара $(B, r)$ обозначается через $\exp ((A, c), m)$. Нетрудно проверить, что

$$
\forall A \in S_{D}, c \in \mathbf{R}, n, m \in \mathbf{N}: \exp (\exp ((A, c), m), n)=\exp (\exp ((A, c), n), m) .
$$

Это свойство введенной операции экспоненцирования и позволяет естественным образом строить различные криптографические протоколы, стойкость которых определяется сложностью проблемы обращения операции экспоненцирования. В [14] доказывается, что к этой проблеме с помощью вероятностного полиномиального алгоритма сводится проблема факторизации целых чисел.

В частности, авторы предлагают протокол открытого распределения ключей типа Диффи-Хеллмана и протокол цифровой подписи типа ЭльГамаля. Оценивается и сложность реализации основных операций по реализации таких протоколов. Показано, что и операция редуцирования, и операция экспоненцирования имеют кубическую сложность.

Реализация криптосистем, использующих вещественные квадратичные поля, является более сложной по сравнению со схемами на мнимых квадратичных полях в связи с тем, что в каждом классе эквивалентных идеалов поля $\mathbf{Q}(\sqrt{D})$ при $D<0$ может содержаться большое число редуцированных идеалов. Поэтому вполне естественной является задача отыскания таких полей $\mathbf{Q}(\sqrt{D})$ при $D>0$, для которых каждый класс идеалов содержал бы возможно меньшее число редуцированных идеалов. С этой целью в работе [63] предлагается использовать поля $\mathbf{Q}(\sqrt{D})$, в которых $D$ есть свободное от квадратов число вида $n^{2}+1$ при $n>2$. Доказывается, что для такого поля число $k$ редуцированных идеалов в каждом классе имеет порядок $O(\ln d)$, где $d$, как и ранее, есть дискриминант поля. Заметим, что в общем случае

$$
\frac{2 R_{d}}{\ln d} \leq k \leq \frac{2 R_{d}}{\ln 2}, \quad R_{d}=O(\sqrt{d} \ln d) .
$$

Учитывая, что в рассматриваемом автором случае число $k$ сравнительно мало́, он предлагает упорядочить редуцированные идеалы $a+b(d+\sqrt{d}) / 2$ по лексикографическому расположению пар $(a, b)$ и выбирать минимальный 
идеал, например, перебором всех редуцированных идеалов соответствующего класса.

Для обеспечения криптографической стойкости предлагаемой автором схемы Эль-Гамаля необходимо, чтобы число классов $h(d)$ было достаточно большим и имело достаточно большой простой делитель.

Автором проделано большое число экспериментов, которые подтверждают, что указанные свойства выполняются с большой вероятностью. В частности, при простых и достаточно больших $D=n^{2}+1$ числа $h(d)$ получаются менее гладкими по сравнению с соответствующими числами в мнимых квадратичных полях. В связи с этим автор делает вывод, что использование в схемах Эль-Гамаля простых дискриминантов вида $n^{2}+1$ может обеспечивать стойкость не хуже, чем использование мнимых квадратичных полей.

В 2000 г. появилась работа [25], в которой предлагается схема идентификации, основанная на использовании вещественных квадратичных полей с большим регулятором. Эта схема является аналогом известной схемы идентификации Фиата-Шамира.

В схеме Фиата-Шамира доказывающий (Д) и проверяющий (П) предварительно выбирают открыто две конечные абелевы группы $G, H$, гомоморфизм $\varphi: G \rightarrow H$ и натуральное число $k$. Кроме того, Д в качестве своего секретного ключа выбирает набор $\left(g_{1}, \ldots, g_{k}\right)$ элементов из $G$ и объявляет открытым ключом набор $\left(h_{1}, \ldots, h_{k}\right)$ элементов из $H$, где $h_{i}=\varphi\left(g_{i}\right), i=1, \ldots, k$. Далее партнеры действуют по следующему протоколу.

1. Д выбирает случайно $g \in G$, вычисляет $h=\varphi(g)$ и высылает $h$ проверяющему П.

2. П выбирает случайно набор $e=\left(e_{1}, \ldots, e_{k}\right) \in\{0,1\}^{k}$ и высылает его доказывающему Д.

3. Д вычисляет $r=g g_{1}^{e_{1}} \ldots g_{k}^{e_{k}}$ и высылает проверяющему П.

4. П проверяет равенство $h h_{1}^{e_{1}} \ldots h_{k}^{e_{k}}=\varphi(r)$.

При этом вероятность ошибки для проверяющего не превосходит $1-1 / 2^{k}$, а стойкость схемы обеспечивается сложностью задачи нахождения прообразов при гомоморфизме $\varphi$ без знания секретного ключа.

В [25] предлагается в качестве групп $G, H$ и гомоморфизма $\varphi$ использовать мультипликативную группу $F^{*}$ вещественного квадратичного поля $F=\mathbf{Q}(\sqrt{D})$, группу $P$ главных дробных идеалов поля $F$ и естественный гомоморфизм $\varphi: F^{*} \rightarrow P$, определенный формулой $\varphi(a)=a F_{0}$, где $F_{0}=\mathbf{Q}_{0}(\sqrt{D})$. В этом случае проблема обращения гомоморфизма $\varphi$ эквивалентна проблеме главных идеалов: по заданному $\mathbf{Z}$-базисом идеалу кольца $F_{0}$ выяснить, является ли он главным идеалом, и если он - главный, то найти его обра- 
зующий элемент. Авторы доказывают, что эта проблема полиномиально сводится к аналогичной проблеме для редуцированных идеалов, и потому вместо группы $P$ используется множество $P_{0}$ всех редуцированных идеалов. В указанной статье обсуждается вопрос о таком выборе параметров предложенной схемы идентификации, при которых проблема главных идеалов является трудной. Отмечается, что наиболее эффективными методами решения этой проблемы являются (указанные выше в связи с вычислением порядка группы классов) алгоритм «малый шаг-большой шаг» и индексный метод. Асимптотическая сложность этих методов равна соответственно

$$
O\left(R^{1 / 2} d^{o(1)}\right) \quad \text { и } \exp \left(O(\log d \log \log d)^{1 / 2}\right) .
$$

Отсюда, учитывая связи между регулятором $R$, дискриминантом $d$ и числом классов $h(d)$, получаем: для противостояния указанным методам целесообразно выбирать поле так, чтобы дискриминант $d$ был большим, а число классов - маленьким.

Авторы отмечают, что наиболее эффективный на тот период вариант индексного метода был предложен в [49]. Эксперименты по этому методу, проведенные в [34], показали, что его сложность для $d=2^{687}$ совпадает со сложностью факторизации числа $2^{1024}$. Другие примеры на сравнение сложностей этих методов приводятся в следующей таблице:

$\begin{array}{cc}\text { Факторизация } & \text { Проблема главных идеалов } \\ 1024 & 687 \\ 1536 & 958 \\ 2048 & 1208 \\ 3072 & 1665 \\ 4096 & 2084\end{array}$

В общем случае для противостояния методу «малый шаг-большой шаг» авторы указывают один из путей выбора дискриминанта $d$. Используя предположение о справедливости обобщенной гипотезы Римана и эвристические предположения Коэна-Ленстры, авторы доказывают, что если $d$ есть произведение двух простых чисел $p_{1}, p_{2} \equiv 3(\bmod 4)$, то неравенство

$$
R>2^{k_{1}} \ln d
$$

будет выполняться с вероятностью $1-2^{-k_{2}}$ при условиях $k_{1} \geq 160$ и

$$
\frac{\sqrt{d}}{\ln d \ln \ln d}>2^{k_{1}+k_{2}+1} \text {. }
$$

Заметим, что предположения Коэна-Ленстры касаются вероятностей цикличности и некоторых свойств силовских подгрупп нечетной части $\mathrm{Cl}_{0}(d)$ группы классов $\mathrm{Cl}(d)$. Так, например, согласно этим предположениям, вероятность цикличности группы $\mathrm{Cl}_{0}(d)$ примерно равна 0,977575 (cм. [5], 
c. 291). В [5] отмечается также, что предположения Коэна-Ленстры проясняют следующие качественные наблюдения в имеющихся таблицах.

1. Нечетная часть группы классов чаще всего циклическая.

2. Вероятность делимости $h(d)$ на простое число $p$ близка к $1 / p+1 / p^{2}$.

3. $\sum_{p \leq x} h(p) \sim \frac{x}{8}$, где сумма берется по всем простым числам $p \leq x$, сравнимым с 1 по модулю 4.

Из анализа таблиц возникает также гипотеза о том, что регулятор $R(d)$ «обычно» имеет порядок $\sqrt{d}$, а тогда величина $h(d)$ должна быть маленькой, в силу теоремы Брауэра-Зигеля, по которой

$$
\ln (R(d) h(d)) \sim \ln \sqrt{d} .
$$

Пока остается открытой знаменитая гипотеза о бесконечности множества таких чисел $d$, при которых $h(d)=1$.

В заключении работы [34] авторы приводят таблицу затрат времени на выбор основных параметров и на верификацию в предлагаемой ими схеме идентификации при $d=2^{m}, m=687,968,1208,1665,2084, k_{1}=160, k_{2}=80$. При этом ошибка проверяющего может происходить с вероятностью, не превосходящей числа $1-1 / 2^{30}$. Вычисления производились на компьютере Pentium II, 300MHz, 64 MB. В приводимой ниже таблице используются те же обозначения параметров, что и в описанной выше схеме Фиата-Шамира. Время выбора соответствующих параметров указывается в секундах.

$\begin{array}{cccccc}m & 687 & 958 & 1208 & 1665 & 2084 \\ d & 0,98 & 3,96 & 13,25 & 16,16 & 63,42 \\ \text { Ключ } & 54,87 & 84,56 & 122,74 & 196,4 & 291,76 \\ h & 3,23 & 4,86 & 7,03 & 10,7 & 16,03 \\ \text { Проверка } & 3,28 & 4,96 & 7,15 & 11,04 & 16,45\end{array}$

В 2001 г. М. Джекобсон, Р. Шайдлер и Ш. Вильямс в [47] предложили новый вариант криптосистемы типа Диффи-Хеллмана, основанной на использовании множества $S$ главных редуцированных идеалов вещественного квадратичного поля. Предлагаемая ими схема по форме ближе к классической, а также и к схемам, основанным на мнимых квадратичных полях. Разница в том, что в ней используется не группа, а группоид $(S, *)$, где $(*)-$ введенная ранее Шенксом операция умножения идеалов с последующим редуцированием результата.

Схематично идею нового подхода можно описать следующим образом.

Абоненты А, В публично выбирают идеал $I \in S$, случайно выбирают секретные экспоненты $a, b$ и вычисляют редуцированные идеалы, ближай- 
шие к $I^{a}, I^{b}$, соответственно. Общим ключом будет идеал $J \in R_{\Delta}$, ближайший к идеалу $I^{a b}$.

Для реализации этой схемы авторы вводят понятие $(f, p)$ представления главного примитивного идеала $I$ при $f \in \mathbf{R}, p \in \mathbf{N}$, удовлетворяющих условию $1 \leq f<2^{p}$. Под этим понимается пара $(J, d)$, где $J-$ идеал, эквивалентный $I, d \in \mathbf{N}$ и $d$ удовлетворяет условию

$$
\text { «Если } \alpha \in K \text { и } J=(\alpha) I \text {, то । }\left(2^{p} \alpha / d\right)-1<f 2^{-p} » \text {. }
$$

Если при этом идеал $J$ является редуцированным, то представление $(f, p)$ также называется редуцированным. Отсюда следует, что $\mid \theta-d 2^{-p}<f d 2^{-2 p}$, и значит, число $d 2^{-p}$ является приближением числа $\theta$ с точностью до $f d 2^{-2 p}$.

В работе развивается арифметика $(f, p)$ представлений идеалов, указываются алгоритмы умножения, возведения в степень и редуцирования $(f, p)$ представлений примитивных идеалов. На основе этих операций и строится протокол выработки ключа абонентами А, В.

Одно из преимуществ указанной схемы выработки ключа заключается в том, что при ее реализации все вычисления можно вести с точностью до $p$ бит, если $2^{p}>46 H^{2} \log _{2} H$ (вместо $2^{p} \geq 3072 \sqrt{D} H^{2}$ в ранее предлагавшихся схемах).

В 2006 г. те же авторы в работе [48] предложили более простую реализацию протокола из [47]. Во-первых, они обратили внимание на то, что в определении $(f, p)$ представления идеала не накладываются ограничения на используемый параметр $d$. В связи с этим ими было дано новое, уточненное, определение $(f, p)$ представления. А именно, $(f, p)$ представлением идеала $I$ при заданных $p \in N, f \in R, 1 \leq f<2^{p}$ они назвали тройку $(J, d, k)$, в которой $J-$ идеал, эквивалентный $I, d \in N, 2^{p}<d \leq 2^{p+1}, k \in Z$ и существует $\alpha \in K$ такое, что $J=(\alpha) I$ и I $\left(2^{p-k} \alpha / d\right)-1 k f 2^{-p}$. Во-вторых, они воспользовались идеей Шенкса из [69], заменили ряд сложных этапов в реализации схемы применением расширенного алгоритма Евклида.

В итоге практическая реализация схемы стала значительно проще, чем ее реализация из статьи [47], хотя теоретическая асимптотическая оценка сложности осталось той же. Для сравнения авторы приводят экспериментально полученную таблицу временных затрат в секундах при выработке 
ключа по рассмотренным выше методам, а также и в сравнении с выработкой ключа в схеме, основанной на мнимом квадратичном поле.

$\begin{array}{ccccc}\log _{2} \Delta & m & r_{1} & r_{2} & r_{1} / r_{2} \\ 795 & 0,04 & 0,38 & 0,13 & 2,8948 \\ 1384 & 0,11 & 1,05 & 0,30 & 3,4518 \\ 1732 & 0,15 & 1,63 & 0,43 & 3,7925 \\ 3460 & 0,50 & 6,34 & 1,45 & 4,3814 \\ 5704 & 1,32 & 17,97 & 3,86 & 4,6553\end{array}$

В этой таблице 1-я строка - входная, под буквами $m, r_{1}, r_{2}$ приводятся данные для мнимого поля и для вещественного поля в алгоритмах из статей [47], [48] соответственно.

О стойкости новой схемы известно лишь следующее.

Из результатов работы [47] следует, что ПДЛ в классе главных редуцированных идеалов не проще проблемы главного идеала, которая формулируется следующим образом. По заданному идеалу $(a, b, c)$ выяснить, является ли он главным, и если да, то найти порождающий его элемент. Известно, что эта проблема не проще проблемы факторизации целых чисел.

Проблема главного идеала в вещественном квадратичном поле является классической. Приведем оценки ее сложности, полученные в последнее время. В 1994 г. К. Абель доказал, что при выполнении обобщенной гипотезы Римана ПДЛ в квадратичном поле $\mathbf{Q}(\sqrt{D})$ при $D>0$ она может быть решена индекс-методом со сложностью $L_{\mid d}(1,144)$.

В 2000 г. У. Вольмер [70] улучшил эту оценку, заменив 1,44 на $\sqrt{2} \approx 1,41$. Более простой для практического вычисления (но с той же асимптотической оценкой сложности) алгоритм предложил М. Джекобсон [50]. Его алгоритм основан на специальном квадратичном решете, применяемом при факторизации чисел. Отметим, что алгоритм решения ПДЛ сложности $\exp (\log D)^{1 / 3}(\log \log D)^{1 / 3}$, которая достигнута для проблемы факторизации целых чисел, к настоящему времени не построен.

\section{Выводы}

Криптосистемы всех рассмотренных типов представляют определенный теоретический и практический интерес. Однако для принятия решения о целесообразности их практического использования и для выбора параметров, обеспечивающих их криптографическую стойкость, необходим дальнейший более тщательный анализ алгоритмов зашифрования, расшифрования и дешифрования в таких системах. 


\section{Список литературы}

1. Боревич 3. И., Шафаревич И. Р. Теория чисел. - М.: Наука, 1964.

2. Гекке Э. Лекции по теории алгебраических чисел. - М.: ГИТТЛ, 1940.

3. Глухов М. М., Пичкур А.Б. Методы алгебраической теории чисел в криптографии. - М., 1998.

4. Ankeny N. C., Artin E., Chowla S. The class-number of real quadratic number fields. - Ann. Math., 1952, v. 56, № 3, p. 479-493.

5. Cohen $H$. A course in computational algebraic number theory. - Berlin: Springer, 1993.

6. Cohen H., Diaz F., Olivier M. Computing ray class groups, conductors and discriminants. - In: ANTS-II, Lect. Notes Comp. Sci., 1996, v. 1122, p. 49-57.

7. Kuroda S. Uber die klassenzahlen algebraischer zahlkorper. - Nagoya Math. J., 1950, v. 1, p. 1-10.

8. Lagarias J. C. Worst-case complexity bounds for algorithms in theory of integral quadratic forms. - J. Algorithms, 1980, v. 1, p. 142-186.

9. Pohst M., Zassenhaus H. Algorithmic algebraic number theory. - Cambridge: Cambridge Univ. Press, 1989.

10. Pohst M. Computational algebraic number theory. - Birkhäuser, 1993.

11. Stefenhagen $P$. The arithmetic of number rings. - MSRI Publ., 2008, v. 44, p. 209-266.

12. Atkin A. O. L. Letter to Shanks on the programs NUDUPL and NUCOMP, 1998 (см. van der Poorten A.J. A note on NUCOMP. - Math. Comp., 2003, v. 72, p. 1935-1946).

13. Biehl J., Buchmann J. A. Algorithms for quadratic orders. — In: Proc. Symp. Appl. Math., 1994, v. 48, p. 425-449.

14. Biehl J., Buchmann J. A., Thiel Ch. Cryptographic protocols based on discrete logarithms in real-quadratic orders. - In: CRYPTO'94, Lect. Notes Comp. Sci., 1994, v. 839, p. 56-60.

15. Biehl J., Buchmann J. A. An analysis of the reduction algorithms for binary quadratic forms. - In: P. Engel and H. Syta «Voronoi`s Impact on Modern Science», v. 1. - Kyiv: Kyiv Institute of Math., National Acad. Sci., 1998.

16. Biehl J., Buchmann J. A., Hamdy S., Meyer A. Cryptographic protocols based on the intractability of extracting roots and computing discrete logarithms. Tech. Rep. - Darmstadt: Technology Univ., 1999.

17. Biehl J., Buchmann J. A., Hamdy S., Meyer A. A signature scheme based on the intractability of computing roots. - Des., Codes and Cryptography, 2002, v. 25 , p. $223-236$.

18. Biehl J., Meyer B., Thiel Ch. Cryptology protocol based on real-quadratic Afields. - In: ASIACRYPT'96, Lect. Notes Comp. Sci., 1996, v. 1163, p. 15-25.

19. Biehl J., Paulus S., Takagi T. An efficient undeniable signature scheme based on non-maximal imaginary quadratic orders. - Tech. Rep. - Darmstadt: Univ.Technology, 1999. 
20. Buchmann J. On the computation of units and class numbers by a generalization of Lagrange algorithms. - J. Number Theory, 1987, v. 26, p. 8-30.

21. Buchmann J. Number theoretic algorithms and cryptology. - In: Proc. FCT'91, Lect. Notes Comp. Sci., 1991, v. 529, p. 16-21.

22. Buchmann J., Dullmann S. On the computation of discrete logarithms in class groups. - In: CRYPTO 90, Lect. Notes Comp. Sci., 1991, v. 537, p. 134-139.

23. Buchmann J., Dullmann S. A probabilistic class group and regulator algorithm and its implementation. - In: Computational number theory. - Berlin-New York: Springer, 1991, p. 53-72.

24. Buchmann J., Dullman H., Williams H. C. On the complexity and efficiency a new key exchange system. - In: EUROCRYPT 89, Lect. Notes Comp. Sci., 1990, v. 434, p. 597-616.

25. Buchmann J., Maurer M., Möller B. Cryptography based on number fields with large regulator. - Tech. Rep. № TI-5, 2000, p. 1-12.

26. Buchmann J., Paulus S. A one way function based on ideal arithmetic in number fields. - In: CRYPTO 97, 1997, p. 385-394.

27. Buchmann J., Scheidler R., Williams H.C. A key exchange system based on real quadratic fields. - In: CRYPTO 89, Lect. Notes Comp. Sci., 1990, v. 435, p. $335-343$.

28. Buchmann J., Williams H.C. A key exchange protocol using real quadratic fields. - J. Cryptology, 1994, № 3, p. 171-199.

29. Buchmann J., Williams H. C. A key exchange system based on imaginary quadratic fields. - J. Cryptology, 1988, № 1, p. 107-118.

30. Dullman S. Ein neues Verfahren zum offentlichen Schusselaustausch. Master thesis. - Dusseldorf: Dusseldorf Univ. Press, 1988.

31. Estes D., Adleman L. M., Kompella K., McCurley K. S., Miller G. L. Breaking the Ong-Schnorr-Shamir signature scheme for quadratic number fields. In: CRYPTO'1985, Lect. Notes Comp. Sci., 1986, v. 218, p. 3-13.

32. Hafner J. L., McCurley K. S. A rigorous subexponential algorithm for computation of class groups. - J. Amer. Math. Soc., 1989, v. 2, p. 837-850.

33. Hamdy S. The key-length of DL-based cryptosystems in class group. - Manuscript, 1999.

34. Hamdy S., Maurer M. Feige-Fiat-Shamir identification based on real quadratic fields. - Technischer Bericht TU-Darmstadt, 2000.

35. Hamdy S., Möller B. Security of cryptosystems based on class groups of imaginary quadratic orders. - In: ASIACRYPT'2000, Lect. Notes Comput. Sci., 2000, v. 1976, p. 234-247.

36. Hamdy S. Performance and security of cryptosystems based on class groups of imaginary quadratic orders. - Manuscript, 2000.

37. Hartmann M., Paulus S., Takagi T. NICE - New Ideal Coset Encryption. In: CHES'99, Lect. Note. Comp. Sci., 1999, v. 1717, p. 341-352. 
38. Hühnlein D. A survey of cryptosystems based on imaginary quadratic orders. 2000, 15 pp. http://citeseerx.ist.psu.edu/.

39. Hühnlein D. Quadratic orders for NESSIE - overview and parameter sizes of three public key families. — 2001, 19 pp. http://citeseerx.ist.psu.edu/.

40. Hühnlein D. Efficient implementation of cryptosystems based on non-maximal imaginary quadratic orders. - Lect. Notes Comp. Sci., 2000, v. 1758, p. 147-162.

41. Hühnlein D., Jacobson M. J., Paulus S., Takagi T. A cryptosystem based on non-maximal quadratic orders with fast decryption. - In: EUROCRYPT'98, Lect. Notes Comp. Sci., 1998, v. 1403, p. 294-307.

42. Hühnlein D., Merkle J. An efficient NICE-Schnorr-type cryptosystem. Lect. Notes Comp. Sci., 2000, v. 1751, p. 328-339.

43. Hühnlein D., Meyer A., Takagi T. Rabin and RSA analogues based on nonmaximal imaginary quadratic orders. - In: Proc. CICS'98, 1998, p. 221-240.

44. Hühnlein D., Paulus S. On the implementation of cryptosystems based on real quadratic number fields. - In: $7^{\text {th }}$ Ann. Workshop Select. Areas Cryptography, Lect. Notes Comp. Sci., 2001, v. 2012, p. 288-302.

45. Hühnlein D., Takagi T. Reduction logarithms in totally non-maximal quadratic orders to logarithms in finite fields. - In: ASIACRYPT'99, Lect. Notes Comp. Sci., 1999, v. 1716, p. 220-231.

46. Jacobson M. J.(Jr.). Computing of discrete logarithms in quadratic orders. J. Cryptology, 2000, v. 13, No 4, p. 437-492.

47. Jacobson M. J.(Jr.), Scheidler R., Williams H. C. Efficient and security of a real quadratic field based-key exchange protocol. - In: Public-Key Cryptography and Comput. Number Theory. - Berlin, 2001, p. 89-112.

48. Jacobson M. J.(Jr.), Scheidler R., Williams H. C. An improved real quadratic field based key exchange procedure. - J. Cryptology, 2006, v. 19, № 2, p. 211-239.

49. Jacobson M. J.(Jr.), Sawilla R. E., Williams H. C. Efficient ideal reduction in quadratic field based-key exchange protocol. - J. Comp. Sci., 2006, № 1, p. 83-116.

50. Jacobson M. J.(Jr.) Subexponential class group computations in quadratic orders. — Ph. D. thesis. Darmstadt, 1999.

51. Kaplan P., Williams K. S. The distance between ideals in the orders of a real quadratic field. - Enzeign. Math., v. 36, No. 3-4, p. 321-358.

52. McCurley $K$. A key distribution system equivalent to factoring. - J. Cryptology, 1988, v. 1, p. 95-105.

53. McCurley K. Cryptology key distribution and computation in class groups. Number Theory Appl., ser. C, 1989, v. 265, p. 459-479.

54. Meyer A. Ein neues Identifications- und Signaturverfahren uber imaginarquadratischen Zahlkorpern. - Master Thesis. - Saarbrucken: University of Saarbrucken, 1997. 
55. Mollin R. A., Williams H. C. Computation of the class number of a real quadratic field. - Util. Math., 1992, v. 41, p. 59-308.

56. Oesterle J. Nombre de classes des corps quadraticues imaginarires. - Soc. Math. De France, 1985, p. 309-323.

57. Ong H., Schnorr C. P., Shamir A. An efficient signature scheme based on quadratic forms. - In: Proc. $16^{\text {th }}$ ACM Symp. Theory of Computing, 1984, p. 208-216.

58. Paulus $S$. An algorithm of subexponential type computing the class group of quadratic orders over principal ideal domains. - Lect. Notes Comp. Sci., 1996, v. 1122, p. 243-257.

59. Paulus $S$., Takagi T. A new public-key cryptosystem over the quadratic order with quadratic decryption time. - J. Cryptology, 2000, v. 13, p. 263-272.

60. Rickert N. W. Efficient reduction of quadratic forms. - In: Computers and Math. - N.Y.: Springer, 1989, p. 135-139.

61. Sawilla R. E. Fast ideal arithmetic in quadratic fields. - Master Thesis. Calgary, Calgary Univ. Press, 2004.

62. Scheidler R., Buchmann J., Williams H. C. Implementation of a key exchange protocol using some real quadratic fields. - In: EUROCRYPT'90, Lect. Notes Comp. Sci., 1991, v. 473, p. 98-109.

63. Scheidler R., Buchmann J., Williams H. C. A key-exchange protocol using real quadratic fields. - J. Cryptology, 1994, v. 7, p. 171-199.

64. Schielzeth D., Pohst M. E. On real quadratic number fields suitable for cryptography. - J. Exper. Math., 2005, v. 14, № 2, p. 189-197.

65. Schönhage A. Fast reduction and composition of binary quadratic forms. In: On Symbolic and Algebraic Computation, ACM, 1991, p. 128-133.

66. Schoof R. J. Quadratic fields and factorization. - In: Computational Methods in Number Theory. Part II. - Amsterdam: Math. Centrum Tracts, 1983, p. $235-286$.

67. Seysen $M$. A probabilistic factoring algorithm with quadratic forms of negative discriminant. - Math. Comp., 1987, v. 48, p. 737-780.

68. Shanks D. Class number, a theory of factorization and genera. - In: Proc. Symp. Pure Math. - AMS, 1971, v. 20, p. 415-440.

69. Shanks $D$. The infrastructure of real quadratic fields and its application. - In: Proc. 1972 Number Theory Conf. - Colorado: Boulder, 1973, p. 217-224.

70. Vollmer $U$. Asymptotically fast discrete logarithms in quadratic fields. - In: ANTS'2000, Lect. Notes Comp. Sci., 2000, v. 1838, p. 581-594.

71. Weber D. Computing discrete logarithms with quadratic number rings. - In: EUROCRYPT'97, Lect. Notes Comp. Sci., 1997, v. 1233, p. 171-183.

72. Williams $H$. A modification of the RSA public-key encryption procedure. IEEE Trans. Inf. Theory, 1980, v. IT-26, p. 726-729.

73. Williams $H$. Some public-key crypto-functions as intractable as factorization. Cryptologia, 1985, v. 9, № 3, p. 223-237. 